http://sciforum.net/conference/ece-1

Conference Proceedings Paper - Energies ,, Whither Energy Conversion? Present Trends, Current Problems and Realistic Future Solutions"

\title{
Monolithic Solar Water-Splitting Systems: Towards a Sustainable Hydrogen-Energy Future
}

\author{
Andrés G. Muñoz* \\ 1 Gesellschaft für Anlagen und Reaktorsicherheit (GRS)mbH, Theodor-Heuss-Straße 4, 38122 \\ Braunschweig, Germany; andres.munoz@grs.de \\ * Author to whom correspondence should be addressed; andres.munoz@grs.de; \\ Tel.: +49-531-8012-240; Fax: +49-531-8012-200.
}

Received: 15 January 2014 / Accepted: 13 March 2014 / Published: 14 March 2014

\begin{abstract}
On of challenging routes to convert solar energy in storable fuels is the lightinduced water-splitting on integrated tandem-systems based on the assembly of stable and efficient semiconducting photoelectrodes. In an inorganic approach, efficient photoelectrodes are pursued by embedding electro-catalytic centers in a passivated semiconductor substrate having an extremely low concentration of surface reaction sites. Condition (i) requires high absorptivity of the semiconducting material, whereas condition (ii) requires control of the electronic properties of the various interfaces and (iii) implies a better understanding and steering of the electro-catalytic process occurring at the surface of reactive centers that convert sunlight directly to fuels. The future implementation of surface modified materials into tandem structures is discussed and future directions concerning the exploitation of photonic effects at metal arrangements of plasmonic materials and the implementation of bio-electrocatalysts in advanced devices is outlined.
\end{abstract}

Keywords: keyword; keyword; keyword (3-10 keywords separated by semi colons)

\section{Introduction}

Innovative solar water splitting systems based on semiconducting photoelectrodes are put more and more in the focus of the research activities as a clean source of fuels. Presently, two major routes for water splitting are considered: the use of highly efficient photovoltaic solid state devices combined 
with electrolysers [1] and the development of monolithically integrated structures that convert sunlight directly to fuels $[2,3]$. The former is thought as energy storage method to flatten strong fluctuations of current from wind generation. The latter route is characterized by two general approaches, for example, single photon electrolysis using abundant materials [4-7] and multiple photon electrolysis based on tandem structures [8]. The former includes developments towards artificial photosynthesis [9] and the latter is based on third generation solar energy conversion processes [10] exploiting the excess energy of solar photons.

The operation efficiency of tandem system depends on the adequate coupling of the photocurrentphotovoltage characteristics of constituting photoelectrodes, which define their individual performance. Efficient photoeletrodes based on semiconductor/metal-contacts require i) efficient light harvesting including the excess photonic energy, ii) low recombination losses and iii) high electron transfer rates of photo-generated minority charge carriers at the electrolyte interface. These preconditions are pursued in a system consisting essentially of catalytic centers embedded in a passivated semiconductor substrate having an extremely low concentration of surface reaction sites. Condition (i) requires high absorptivity, whereas condition (ii) requires control of the electronic properties of the various interfaces and (iii) implies a better understanding and steering of the electrocatalytic process occurring at the surface of reactive centers

In this paper, the main electronic und energetic aspects of the conversion process in this type of systems are discussed and exemplified on the basis of preliminary and model experiments concerning the use of technologically advanced IV and III-V semiconductors for half-cell configurations. The future implementation of surface modified materials into tandem structures is discussed and future directions concerning the exploitation of photonic effects at metal arrangements of plasmonic materials and the implementation of bio-electrocatalysts in advanced devices is outlined.

\section{Conversion efficiency: thermodynamic and kinetic aspects}

The principle of working of tandem water splitting systems is outlined in the energy band diagram depicted in fig.1. Light generated electron-hole pairs in each of the constituting semiconductor are separated by an electric field underneath $p n$-junctions or semiconductor-metal catalyst contacts (1). Minority charge carriers are transported to the catalytic metal islands (2), where they are consumed by the electrochemical reactions (3): proton reduction at the photocathode and water oxidation at the photoanode. But a part of photo-generated minority charge carriers is lost by recombination at generated interfaces (4) via electron traps, related with the particular electronic and atomic structure of the interface. In electronic equilibrium, the position of the Fermi level is fixed by the ohmic contact of the constituting p- and n-type semiconductors. This, in turn, determines the maximal achievable photopotential $\left(V_{\mathrm{ph}}\right)$ of each constituting photoelectrode. The condition sine qua non for the working of a tandem system is given by:

$$
V_{p h, p-t y p e}+V_{p h, n-t y p e}=\frac{\Delta G_{r}}{e}+\eta_{a}+\eta_{c}+\Delta V_{\Omega}
$$


where $\Delta G_{r}=1.229 \mathrm{eV}$ is the minimum work for water splitting. $\eta_{\mathrm{c}}(\sim 0.1 \mathrm{~V})$ and $\eta_{\mathrm{a}}(\sim 0.4)$, represents the electrochemical overpotentials related with the kinetics of hydrogen evolution and water oxidation respectively. $\Delta V_{\Omega}$, the ohmic overpotential, arises from resistive losses in the cell and in the residual band bending in the absorber to drive the reaction. These factors lead to a rather pronounced increase of the required $V_{p h}$. The energetic requirements delimit the frame for the selection of the photoelectrode. Thus, semiconductors with a conduction band above the level for proton reduction are suited for the construction of photocathodes, such as GaAs or InP. Materials with a valence band below the level for water oxidation suit for photoanodes, such as $\mathrm{TiO}_{2}, \mathrm{Nb}_{2} \mathrm{O}_{5}$ or $\mathrm{WO}_{3}$.

Figure 1. Energy band diagram showing the working principle of a tandem water splitting device. Detail: schematic of a tandem water splitting cell.
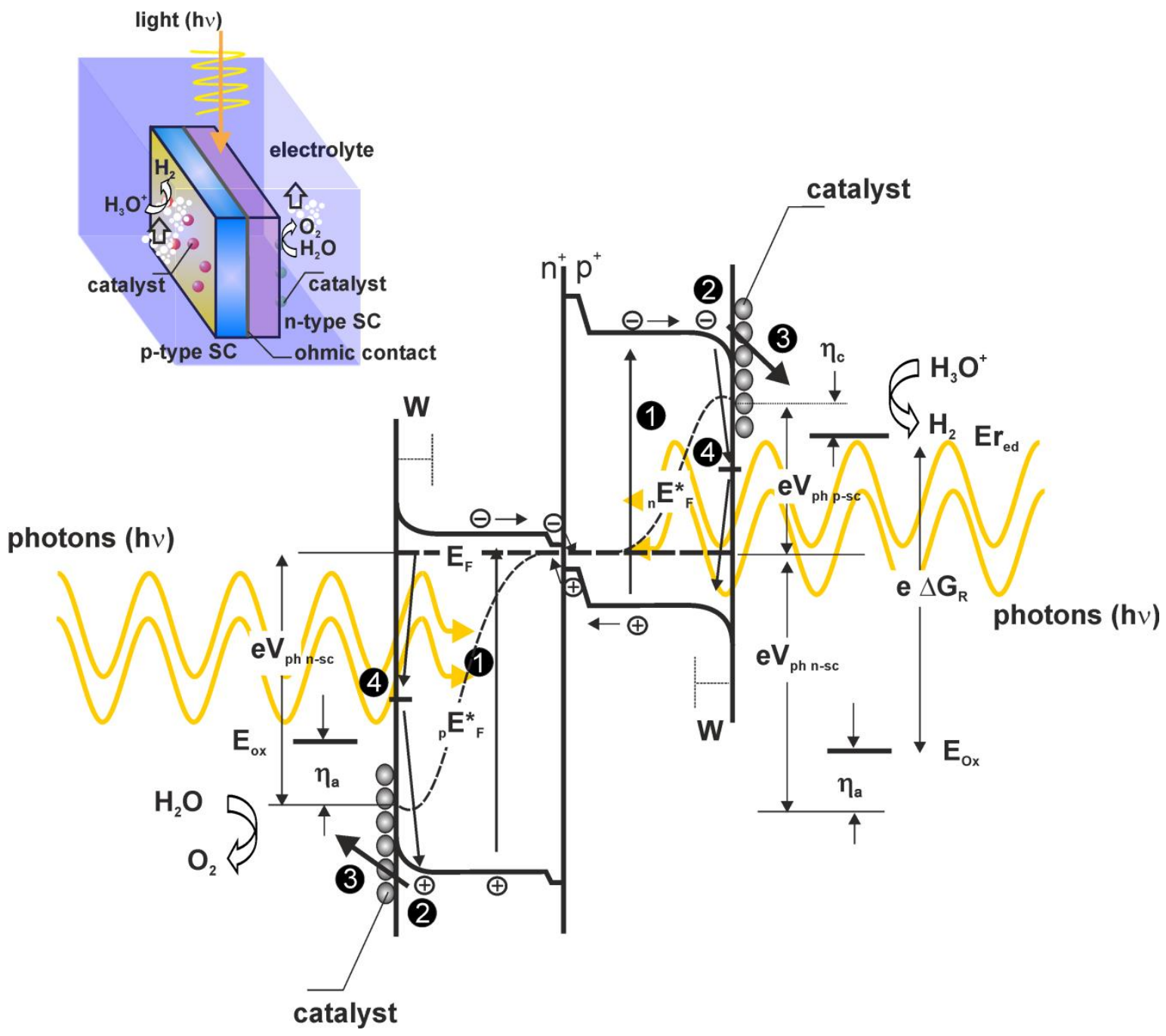

The energy of semiconductor bands for common materials used in the construction of photovoltaic and photoelectrochemical cells are presented in fig. 2 in comparison with the energy levels for the proton reduction (HER) and oxygen evolution reactions (OER). The band gap of semiconductors defines the efficiency of solar light absorption. Materials with a band gap between 1.0 and $1.5 \mathrm{eV}$ yield the maximal achievable absorption efficiency according to the Schockley-Queisser limit [11]. This also limits the maximal achievable current yield. Oxide 
semiconductors are sometimes, in spite of their large band gap, considered as adequate anode materials due to their low preparation costs. The low current yield of photoactive oxides could, however, constitute a bottleneck for the performance of system, which could be circumvented by extending the active by nano-structuring.

Figure 2. Energy bands of some photovoltaic materials. The energy level of electrochemical reactions are referred to an energy for the standard hydrogen electrode of $4.5 \mathrm{eV}$.

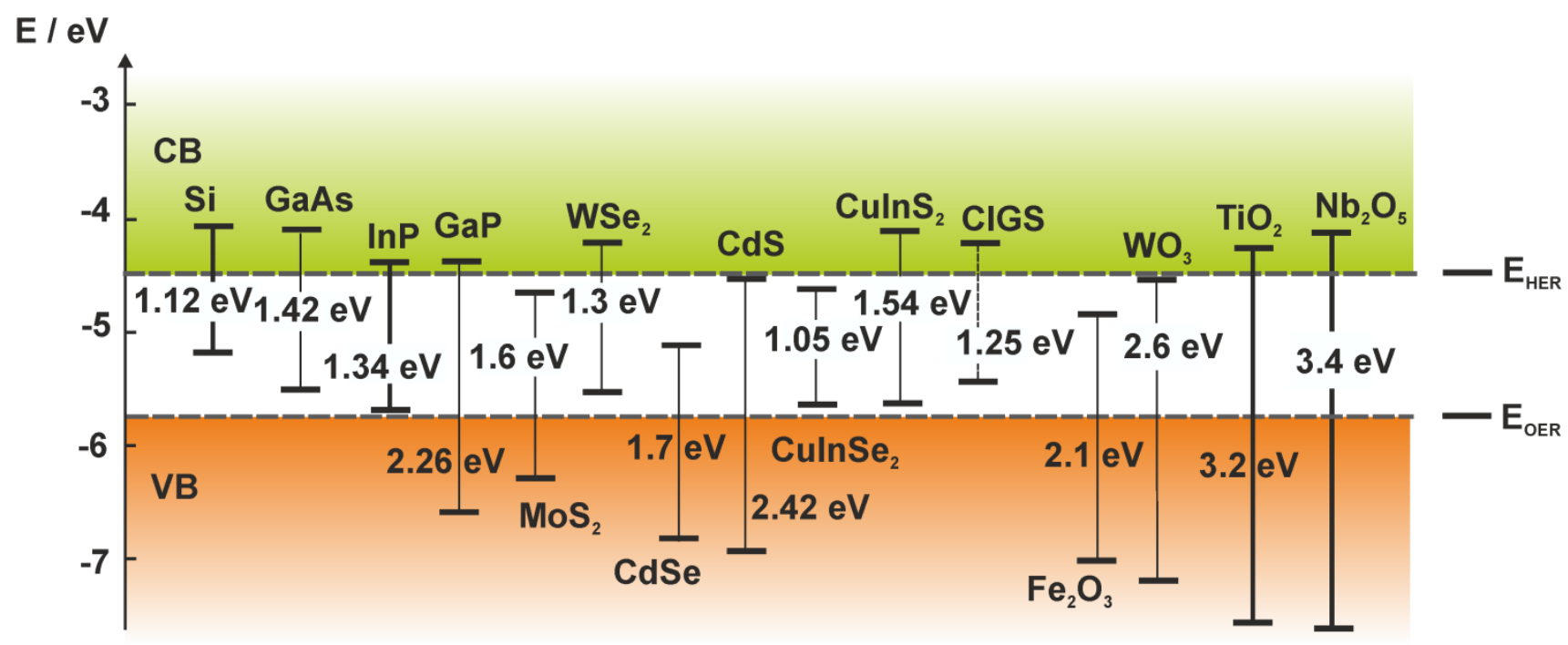

The efficiency of water splitting devices can be expressed as:

$$
\xi=\frac{\dot{m}_{H_{2}} \times \Delta G_{r}}{\int_{0}^{\infty} I(\lambda) d \lambda}
$$

where $\dot{m}_{H_{2}}$ is the flux of generated hydrogen and $I(\lambda)$ is the spectral solar irradiance. The conversion efficiency is determined by the absorption (and reflection) properties, recombination losses and by the rate of electron transfer at the respective semiconductor/electrolyte interfaces. Recombination processes occur at interface states in the energy gap, whose concentration and energy distribution depend on the particular surface nano-topology and chemistry [12]. An efficient electron transfer depends on the nature and on the particular electronic surface properties of the catalyst. The latter is determined by the surface crystalline orientation and by the morphological and structural aspects. The design and development of photoelectrodes is thus based on a maximizing of the individual performance, which is investigated by measuring the current-potential characteristic in a threeelectrode photoelectrochemical cell. Fig. 3 shows a qualitative representation of the characteristics of individual tandem photoelectrodes. The blue curves represent the current-voltage relations for the metal catalysts in the dark. The rate of water splitting is determined by the coupling current at the crossing point of photoelectrode characteristics $\left(J_{\mathrm{op}}, V_{\mathrm{op}}\right)$, i.e. the internal current. 
Figure 3. Qualitative description of current-voltage curves of photoelectrodes in tandem systems.

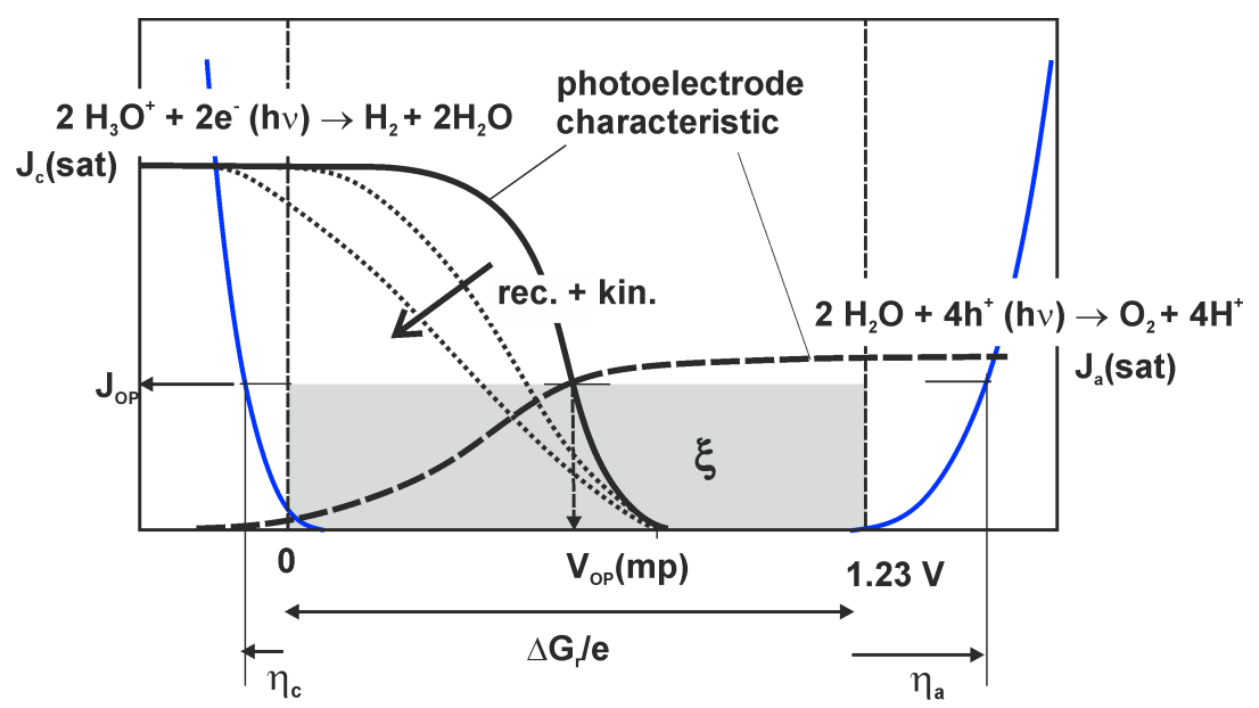

The saturation photocurrent $J_{\mathrm{a}, \mathrm{c}}(\mathrm{sat})$ depends on the reflection and adsorption coefficient of the semiconductor. The raising part of the curves is determined essentially by the transfer of photogenerated minority charge carriers from the semiconductor into the electrolyte. Recombination losses and poor electrochemical kinetics flatten the curve with a consequent loss of efficiency (dotted curves). The overvoltage necessary to drive the electrochemical reactions $\left(\eta_{\mathrm{a}}, \eta_{\mathrm{c}}\right)$ is that given by the current-voltage characteristics of the electrochemical catalysts (blue curves).

The photoelectrochemical current-voltage characteristics can be modelled by combining the electrochemical kinetics of metal electrodes with the $I-V$ relation of illuminated diodes. Fig. 4 shows an equivalent circuit of a metal-covered type photoelectrode. The recombination processes are represented by a parallel connected resistance. Ideal photoelectrodes corresponds to $R_{\text {rec }} \rightarrow \infty$. The photovoltaic part of the photoelectrode supplies a current $J_{\mathrm{e}}$ at a voltage $V_{0}$, which is equivalent to the light induced photopotential, $V_{\mathrm{ph}, \mathrm{n}, \mathrm{p}-\mathrm{typ}}=\mid E_{\mathrm{F}^{-} \mathrm{n}, \mathrm{p}} E_{\mathrm{F}}{ }^{*}$ (s) $\mid$ (see fig.1). For the generation of a current $J_{\mathrm{OP}}$ the photopotential should reach an overvoltage $\eta_{\mathrm{a}, \mathrm{c}}$, so that photogenerated charge carriers can be consumed by the electrochemical reaction. The electrochemical process can be represented by a nonlinear resistance of the form:

$$
R_{r}=\left(\frac{\partial J}{\partial \eta}\right)^{-1}
$$

For electrochemical reactions on metals, a convenient description of the current-voltage curve is given the Butler-Volmer equation:

$$
J_{a, c}=J_{0, a, c} \times \exp \left(\alpha_{a, c} \frac{e}{k T} \eta_{a, c}\right)
$$


where $J_{0, \mathrm{a}, \mathrm{c}}$ is the exchange current density and $\alpha_{\mathrm{a}, \mathrm{c}}$ is the charge transfer coefficient: $0<\alpha_{\mathrm{a}, \mathrm{c}}<1$. For this model it is assumed that the photogenerated charge carriers are ballistic discharged at the quasi-Fermi level ${ }_{\mathrm{n}, \mathrm{h}} E_{\mathrm{F}}{ }^{*}$ at the surface. For thick metal layers, injected electrons can thermalize and lose their energy. For instance, the ballistic electron injection of a kinetic energy of 1-2 eV is limited to the inelastic mean free path of 200-300 nm. Typical thermalisation times in metals are of some hundreds of fs [13-15], which is some order of magnitude faster than the span for electron transfer in electrochemical reactions. Therefore, excess electrons not consumed by the electrochemical reaction in this interval, thermalize and recombinate.

Figure 4. (a) Energy band diagram of a p-type photoelectrode showing main electron transfer processes. (b) Energy band diagram showing the potential distribution at the SC/oxide/metal junction. (c) Equivalent circuit of a photoelectrode.

\section{a}
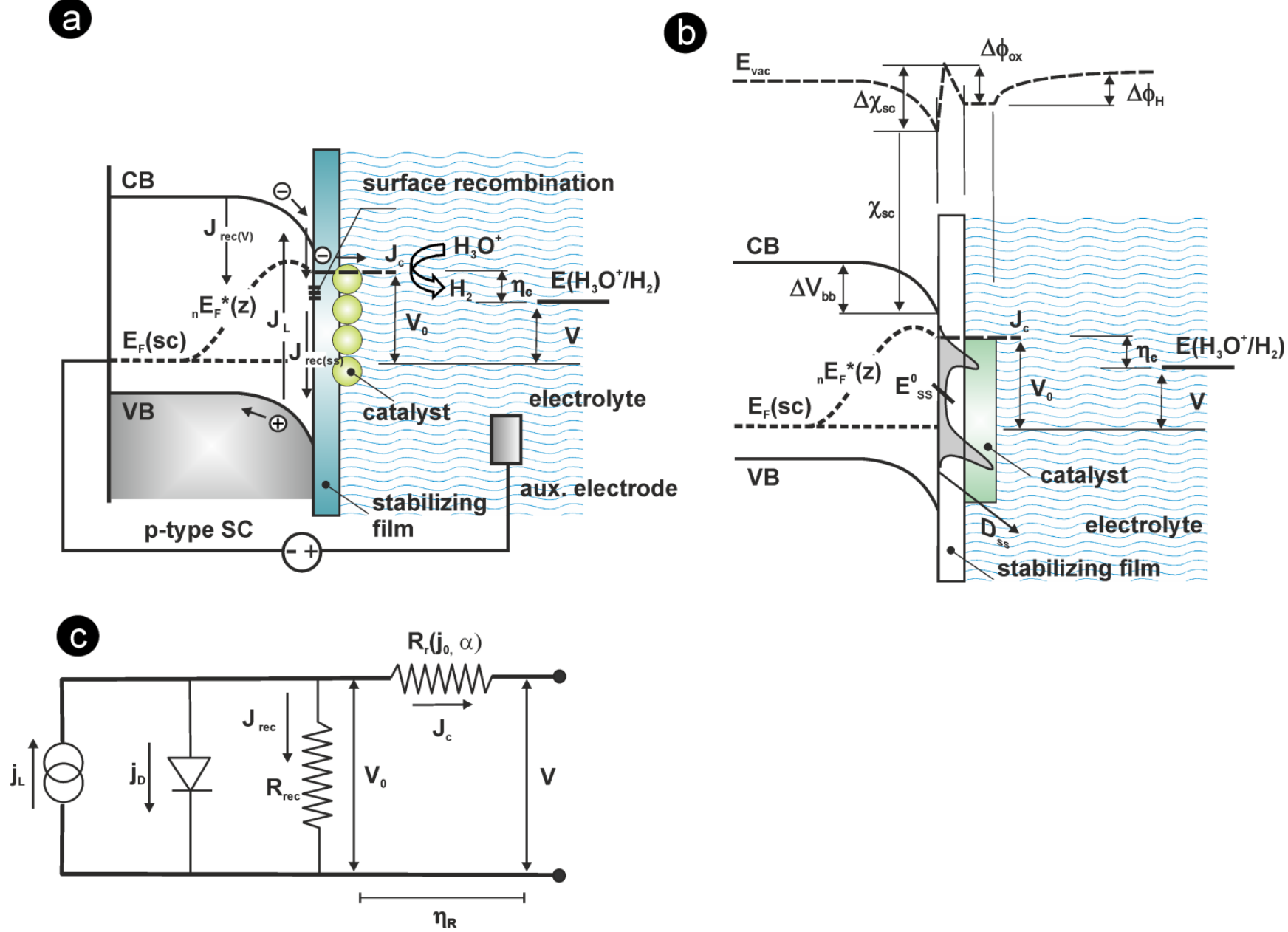

The voltage output of the photoelectrode correspond to that of the photodiode $\left(V_{0}\right)$ reduced by the electrochemical overvoltage $\left(\eta_{\mathrm{a}, \mathrm{c}}\right)$. Thus, the $I-V$ curve can be written as that for a photodiode for a potential $V+\eta_{\mathrm{a}, \mathrm{c}}$ (disregarding recombination effects): 


$$
J_{a, c}=-J_{L}+J_{S}\left[\exp \left(\frac{e}{k T}\left(V+\eta_{a, c}\right)\right)-1\right]
$$

Where $J_{\mathrm{L}}$ is the saturation current of the illuminated photodiode and $J_{\mathrm{S}}$ is the reverse bias saturation current. After elimination of the term $\eta_{\mathrm{a}, \mathrm{c}}$ by combination of equations (4) and (5) we obtain:

$$
V=\frac{k T}{e}\left[\ln \left(\frac{J_{L}-J}{J_{S}}\right)-\frac{1}{\alpha_{a, c}} \ln \frac{J}{J_{0, a, c}}+1\right]
$$

This expression introduced the electrochemical parameters $\alpha_{\mathrm{a}, \mathrm{c}}$ and $J_{0, \mathrm{a}, \mathrm{c}}$, which represent the activity of the used electrocatalyst for a given electrochemical reaction. Fig. 5 shows the influence of these parameters on the current-voltage output of photoelectrodes for material fixed $J_{\mathrm{s}}$ and $J_{\mathrm{L}}$. The catalytic nature of the deposited metal has a large influence on the photoelectrode performance, represented by the colored squares. On the other hand, a drop of efficiency can be observed for $\alpha_{\mathrm{a}, \mathrm{c}}<0.5$.

Figure 5. Influence of electrochemical parameters on photocurrent-voltage curves calculated by eq.(6) for a photocathode (a) catalytic activity of metal (exchange current density, $\mathrm{J}_{0}$ ). (b) Charge transfer coefficient, $\alpha_{\mathrm{c}}$.
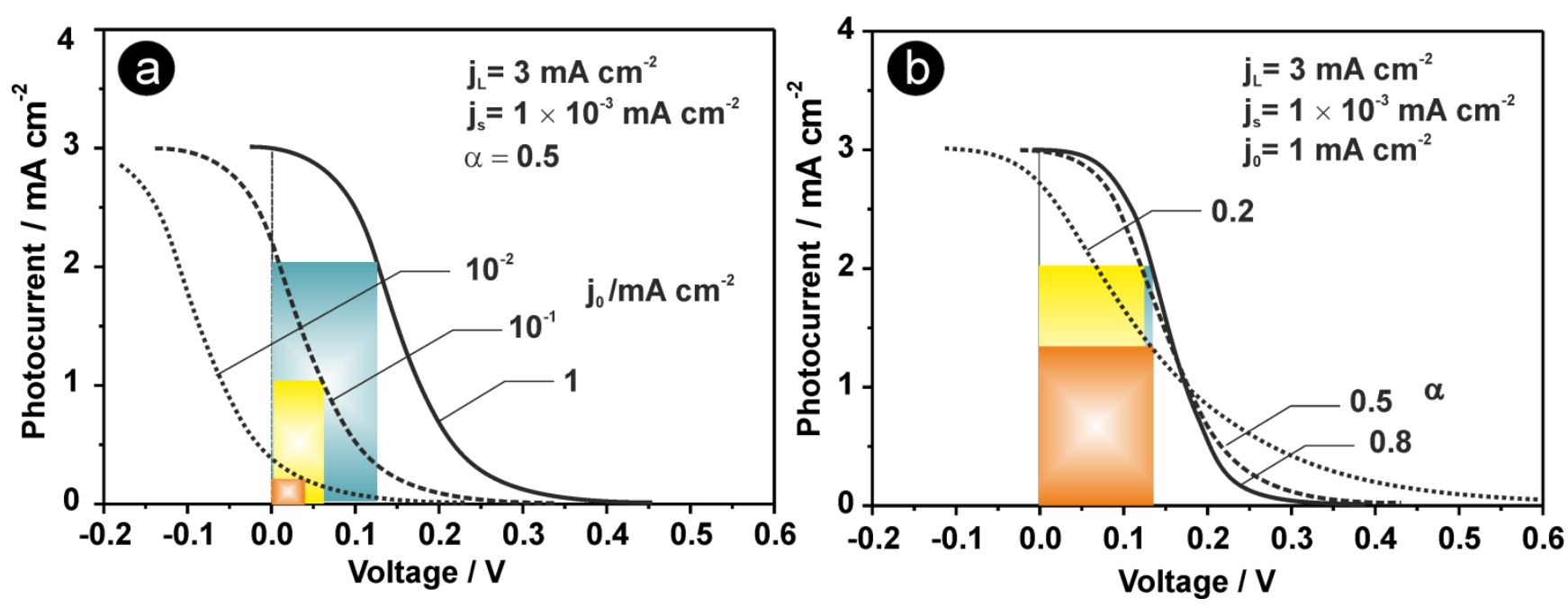

The functionality of the interfacial potential distribution with $V$ is not straightforward and depends on the dependency of ${ }_{n . p} E_{\mathrm{F}}{ }^{*}(\mathrm{~V})$ at the semiconductor surface and the individual variation of the different interfacial potential drops $\left(\Delta \underline{V_{\mathrm{bb}}}\right.$, the semiconductor band bending, $\Delta \phi_{\mathrm{ox}}$, the dielectric potential drop in the interfacial oxide film and $\Delta \phi_{\mathrm{H}}$, the potential drop at the metal/electrolyte interface or Helmholtzlayer) with photoinduced charge accumulation at the surface states (SS). The extent of accumulation depends on the charge carrier dynamics and the density and distribution of surface states at the semiconductor/oxide interface $\left(D_{\mathrm{ss}}\right)[16]$. 


\section{Thin film photoelectrode concepts}

A promising photoelectrode based on the concept of $\mathrm{SC} /$ film/metal structure can be built from thin $\mathrm{p}$ InP homoepitaxial layers deposited by MOVPE (metalorganic vapor phase epitaxy). This type of materials opens a new issue in the fabrication of high efficient photocathodes to be implemented in the construction of monolithic water splitting systems. This concept is technologically feasible, regarding the use of extended liftoff processes [17] for thin films on substrate wafers. A specific process for deposition of In-rich p-type InP with a $(2 \times 4)$ reconstructed surface was implemented [18-20]. The deposited photoelectrode is activated by photoelectrochemical conditioning. It consists of a photoelectrochemical cycling in $\mathrm{HCl}$ solution between $-0.1 \mathrm{~V}$ and $0.3 \mathrm{~V} \mathrm{SCE}$ and subsequent $\mathrm{Rh}$ photoelectrodeposition at $-0.2 \mathrm{~V}$ from $5 \mathrm{mM} \mathrm{RhCl}_{3}+0.5 \mathrm{NaCl}$ in the presence of i-propanol as leveling agent [21]. Fig.6b shows the performance of such an electrode.

Figure 6. (a) HR-TEM picture of a sample cross section of the prepared photoelectrode. (b) output power characteristic of the thin film $\mathrm{p}$-InP device in $1 \mathrm{~mol} / \mathrm{l} \mathrm{HClO}_{4}$. Light intensity: $100 \mathrm{mWcm}^{-2}$ (W-I lamp); open circles: I-V curve of pure Rh; (----) expected I-V characteristic for improved charge transfer.
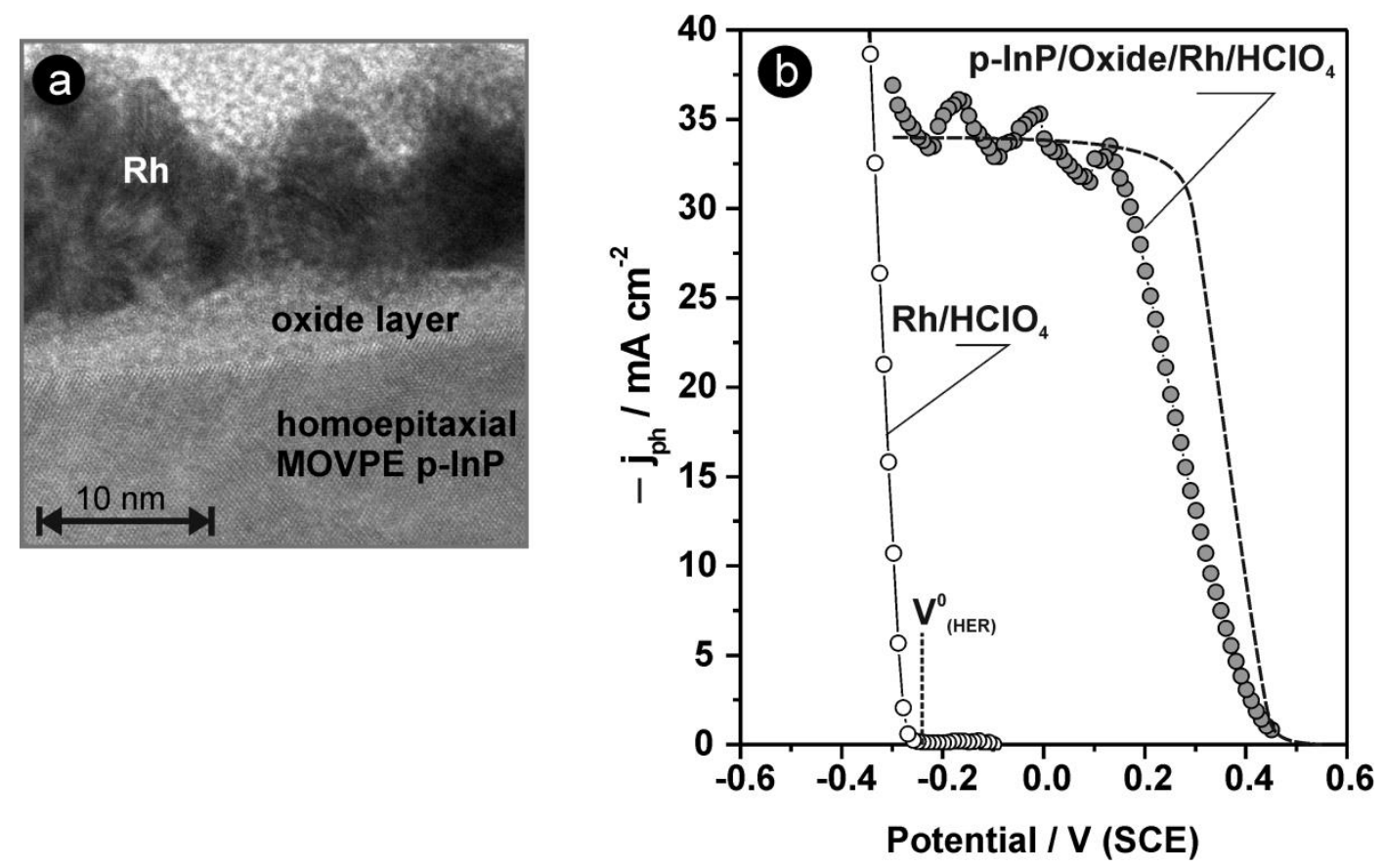

The particular electrochemical activation causes the growth of an amorphous ultra-thin interfacial oxide film onto which a film of interlinked $\mathrm{Rh}$ is formed by galvanic deposition (see fig.6a). Synchrotron radiation photoelectron spectroscopy (SRPES) experiments revealed that this film consists of a complex oxide, the composition of which can be described by the nominal compounds $\mathrm{In}\left(\mathrm{PO}_{3}\right)_{3}, \mathrm{InPO}_{4}$ and $\mathrm{In}_{2} \mathrm{O}_{3} . \mathrm{In}_{2} \mathrm{O}_{3}$ is the predominant structure in the oxide layer [21]. UPS experiments have shown that the formation of the film increases the electron affinity of the InP-absorber, $\chi_{\text {InP }}$ by $\Delta \chi_{\operatorname{InP}}=0.4 \mathrm{eV}: \chi_{\operatorname{InP}}+\Delta \chi_{\operatorname{InP}}=4.35 \mathrm{eV}$. Hence, the modified semiconductor surface reaches a maximal achievable output photopotential of $V_{0}=\chi_{\mathrm{InP}}+E_{\mathrm{g}(\mathrm{InP})}-\Delta E_{\text {doping }}-4.6 \mathrm{eV}(\mathrm{HER})=0.91 \mathrm{eV}$ (see fig.4c). 
Experimentally, the photoelectrode yields a maximal $V_{0} \sim 0.7 \mathrm{eV}$. Also, the photocurrent can still be improved, as shown by the predicted curve in fig.6b. Both effects can be ascribed to the particular electron transfer way across the oxide. Here, the formation of $\operatorname{In}_{2} \mathrm{O}_{3}$-like chemical environments inside the film renders it conductive. Indium oxide is an n-type semiconductor with a band gap of $3.6 \mathrm{eV}$ and an electron affinity of $4.5 \mathrm{eV}$ [22]. Thus, energy terms arise near the conduction band energy of indium oxide, by way of which, electrons can hop. The specific chemical composition and structure of the oxide at the semiconductor surface create electrical dipoles, which allow some kind of band alignment. This indicates a large potentiality of this type of films by varying their surface structure and electrochemical conditioning. This is shown, for instance, by the different $I-V$ characteristics cycled $\operatorname{InP}(111)$ and the reconstructed homo-epitaxial $\operatorname{InP}(100)(2 \times 4)$ film (see fig.7).

Figure 7. Photocurrent-voltage characteristics of different InP-surfaces in $0.5 \mathrm{~mol} / \mathrm{l} \mathrm{HCl}$ after 50 cycles. The details depict the atomic structures of the initial surfaces. White light (I-W lamp) with an intensity of $100 \mathrm{~mW} / \mathrm{cm}^{2}$.

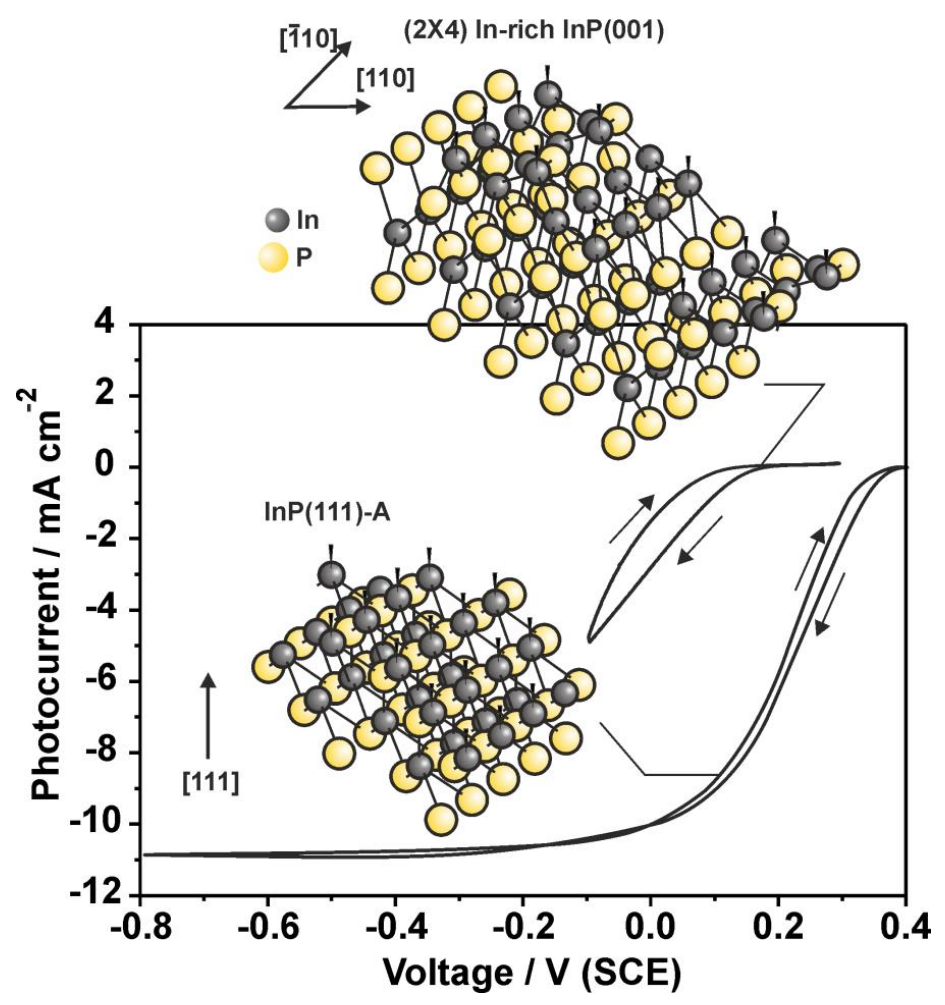

A look ahead in future developments of efficient thin film photoelectrodes let envisage the implementation of multi-junction structures deposited by MOVPE [23]. It is already known from the photovoltaics, that theoretical maximal achievable conversion efficiencies of solar light in electric energy can be increased significantly if the light transmitted through an individual solar cell is absorbed by a subsequent one with a lower band gap. Multi-junction devices constituted by epitaxial subcells connected by a tunnel junction [24] have already shown efficiencies above $40 \%$ under concentrated sunlight [25]. Multi-junction structures combine efficient absorption of the solar spectrum with sufficient voltage for water-splitting devices. Group III-V materials, such as InP, yields world-record solar-to-hydrogen efficiencies [8], but the degradation in contact with the electrolyte 
limits the applicability of such materials. Covering with a homogeneous thin film of an electrocatalytic metal is an option. In another approach, dilute nitride incorporation may improve the stability against electrolytes [26]. Fig.8 shows a contour plot of the limiting efficiency as a function of the band gaps of two-absorber junctions calculated with EtaOpt [27] for the standardized terrestrial solar radiation AM1.5. Although the band gap of silicon $(1.12 \mathrm{eV})$ is above the optimum band value of $0.95 \mathrm{eV}$ for the bottom cell of a tandem-junction, it yields theoretically achievable efficiency of $44.8 \%$ in combination with a $1.73 \mathrm{eV}$ band gap top GaPNAs (indicated with dotted lines in fig.8).

Figure 8. Contour lines of calculated limiting conversion efficiencies for ideal two-film tandem solar cells under AM1.5 irradiation (1000 W, one sun) at ambient temperatures in dependence of the top and bottom cell band.

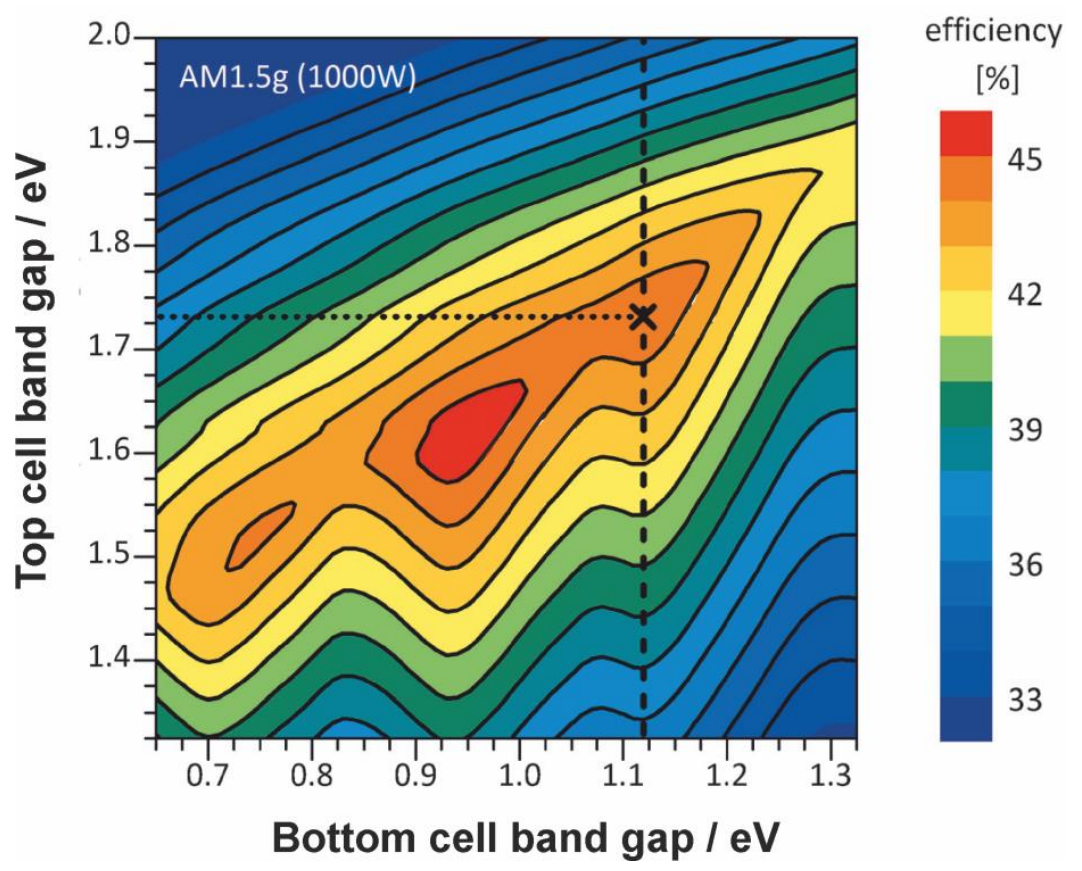

Another approach to enhance the adsorption yield of photoelectrodes bases on the photonic effects in arrangements of nanoparticles of plasmonic materials. This phenomenon arises from confinement of conduction electrons to small particle volumes, resulting in a resonant electromagnetic behavior by scattering of electromagnetic waves. For particles of dimensions $a<<$ wavelength, the conduction electrons inside the particle move all in phase upon plane-wave excitation (see ref. [28] for more details). The coherent electron oscillations are known as localized surface plasmon polaritons. The strength of the dipolar plasmon resonance is quantified by the particle polarizability, given by:

$$
\alpha=4 \pi a^{3} \frac{\varepsilon-\varepsilon_{m}}{\varepsilon+2 \varepsilon_{m}}
$$

where $a$ is the particle radius, $\varepsilon_{\mathrm{m}}$ is the dielectric permittivity of the surrounding medium and $\varepsilon=\varepsilon(v)$ describes the dispersive dielectric response of the metal. The light induced polarization reaches it 
maximum at the Fröhlich frequency [29]. The imaginary part of $\varepsilon$ represents heating losses within the particle, caused by the energy release of induced electron-hole pairs on their coupling with phonons. The periodic arrangements of closely spaced metal nanoparticles convert the optical mode into nonradiating surface plasmons (see fig.9)[30]. The enhanced absorption of light or electromagnetic fields by generation of collective oscillations of free electrons at the metal surface opens new horizons in the photo-electrocatalysis. In principle, metal thin films or adequate arrangement of nanoparticles can be applied to increase the optical path length in thin active photovoltaic layers, thusenhancing the overall photoabsorption [31,32]. The direct influence of electron excitation on the electrochemical process is still unknown. There is some evidence confirming the exchange of electrons from an excited surface of metal particles to chemisorbed molecules, resulting in photochemistry processes. Colloidal Ag nanocrystals stabilized by sodium citrate build up a photovoltage under visible excitation that is caused by irreversible "hot holes" photo-oxidation of adsorbed citrate anion [33]. This creates a driving force for photochemical transformation of round shaped $\mathrm{Ag}$ seeds of $8 \mathrm{~nm}$ into $70 \mathrm{~nm}$ single crystal disk prisms under room light, in a novel type of light-driven Ostwald ripening.

Figure 9. Schematic vision of the generation of surface plasmon polaritons by incident light on an arrangement of nanoparticles of plasmonic metals.

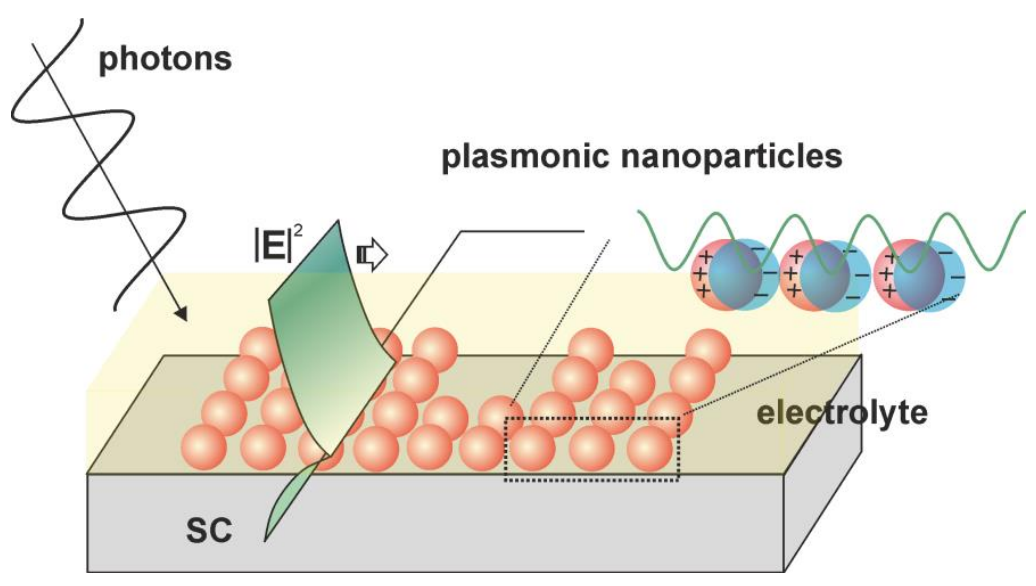

The use of enzymes as bio-inspired electrocatalysts is an approach with increasing acceptability in an attempt to replace the expensive catalytic noble metals. The implementation of enzymes in the construction of in reliable photoelectrodes requires mimicking the active part by eliminating the inherently unstable protein part of natural photosynthetic components. This should also help to accelerate the electron transfer process. The enzymes works generally close to the Nernstian potential and turnover rates as high as $9000 \mathrm{~s}^{-1}[34,35]$. Architectures should consist of mono- and heterometallic cores where the metal atoms are chelated by ligands or organic molecules as a source of free electrons. Artificial photosynthetic systems are presently designed on the basis of Co- und Ni-diiminedioxime and $\mathrm{Ni}-\mathrm{Ru}$ complexes [36], Ni-Fe di-thiolatehydrides [37] as well as Ru-complexes [38], all being classified as excellent catalysts in the dark. A vision of bio-inspired catalytic system is schematized in fig.10. Here, nanoparticle absorbers are assumed that have been deposited on a semiconducting support which would allow rectification, thus using the system in an analogous manner to a photodiode-type solar cell. 
Figure 10. (a) Schematic vision of an hybrid system combining efficient tandem thin film absorbers with bio-inspired electrocatalysts (hydrogenase). (b) Energy diagram representing the electron transfer mode in bio-inspired photoelectrodes.

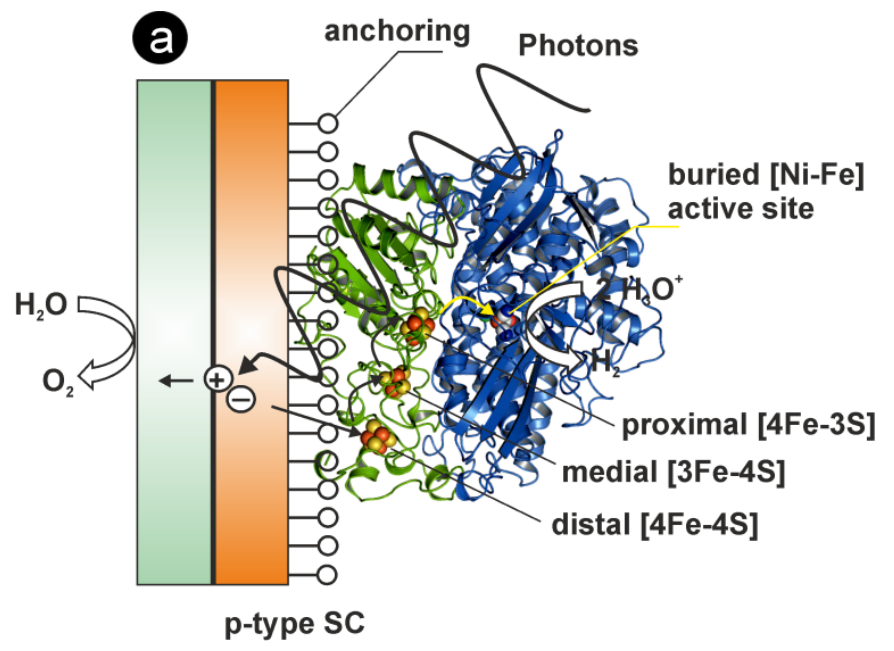

[Fe-Ni] Hydrogenase

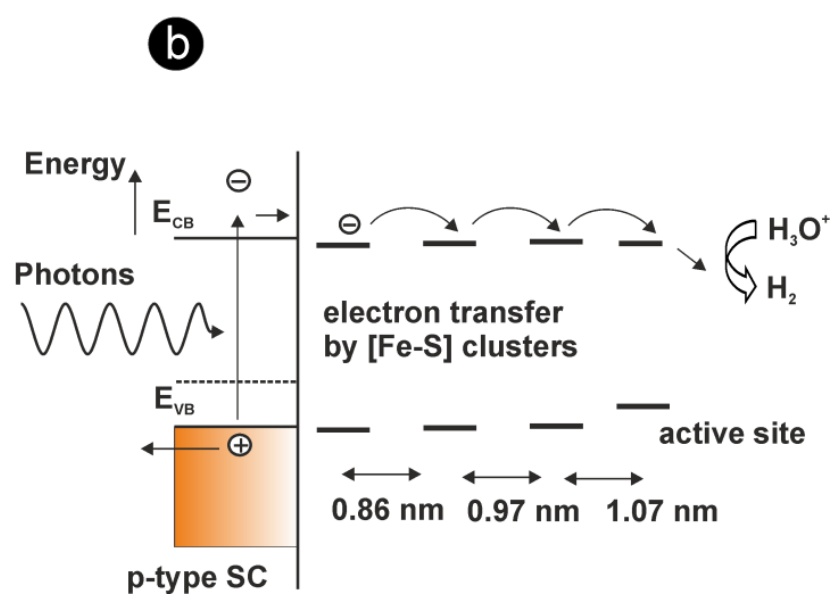

\section{References and Notes}

1. Barbir, F. PEM electrolysis for production of hydrogen from renewable energy sources. Solar Energy 2005, 78, 661-669.

2. Lewis, N. Light work with water. Nature 2001, 414, 589-590.

3. Bard, A.J.; Fox, M.A. Artificial photosynthesis: solar splitting of water to hydrogen and oxygen. Acc. Chem. Res. 1995, 28, 141-145.

4. Aharon-Shalom, E.; Heller, A. Efficient p-InP (Rh- H alloy) and p-InP (Re- H alloy) hydrogen evolving photocathodes . J. Electrochem. Soc. 1982, 129, 2865-2866.

5. Maiera, C.U.; Specht, M.; Bilger, G. Hydrogen evolution on platinum-coated p-silicon photocathodes. Int. J. Hydrogen Energy 1996, 21, 859-864.

6. Tributsch, H. Interfacial processes involving strong electronic interactions in solar energy conversion and storage. J. Photochem. 1985, 29, 89-113.

7. Bak, T.; Nowotny, J.; Rekas, M.; Sorrel, C.C. Photo-electrochemical hydrogen generation from water using solar energy. Materials-related aspects. Int. J. Hydrogen Energy 2002, 27, 991-1022.

8. Khaselev, O.; Turner, J.A. A monolithic photovoltaic-photoelectrochemical device for hydrogen production via water splitting. Science 1998, 280, 425-427.

9. Desai, D.K. Artificial Photosynthesis System: An Engineering Approach. In Artificial photosynthesis: from basic biology to industrial applications; Collings, A.F., Critchley, C., Eds.; Wiley-VCH, Weinheim, 2004, pp. 291-299.

10. Hanna, M.C.; Nozik, A.J. Solar conversion efficiency of photovoltaic and photoelectrolysis cells with carrier multiplication absorbers. J. Appl. Phys. 2006, 100, 074510. 
11. Shockley, W.; Queisser, H.J. Detailed balance limit of efficiency of p-n junction solar cells, $J$. Appl. Phys. 1961, 32, 510.

12. Jaegermann, W. The semiconductor/electrolyte interface: A surface science approach. In Modern Aspects of Electrochemistry; Conway, B.E.; Bockris, J.O’M., Eds.; Plenum Press, New York, 1996; Vol. 30.

13. Guillon, C.; Langot, P.; Del Fatti, N.; Vallée, F. Nonequilibrium electron-loss kinetics in metal clusters. New Journal of Physics 2003, 5, 13.1

14. Del Fatti, N.; Voisin, C.; Achermann, M.; Tzortzakis, S.; Christofilos, D.; Vallée, F. Nonequilibrium electron dynamics in noble metals. Phys. Rev. B 2000, 61, 16956-16966.

15. Sun, C.-K.; Vallée, F.; Acioli, L.H.; Ippen, E.P.; Fujimoto, J.G. Femtosecond-tunable measurement of electron thermalisation in gold. Phys. Rev. B 1994, 50, 15337-15348.

16. Muñoz, A.G.; Lewerenz, H.J. Advances in photoelectrocatalysis with nanotopographical photoelectrodes, ChemPhysChem 2010, 11, 1603-1615.

17. Schermer, J.J.; Bauhuis, G.J.; Mulder, P.; Haver-kamp, E.J.; van Deelen, J.; van Niftrik, A.T.J.; Larsen, P.K. Photon confinement in high-efficiency, thin-film III-V solar cells obtained by epitaxial lift-off. Thin Solid Films 2006, 511-512, 645-653.

18. Hannappel, T.; Töben, L.; Möller, K.; Willig, F. In-situ monitoring of $\operatorname{InP}(100)$ and GaP(100) interfaces and characterization with RDS at 20 K. J. Electron. Mat. 2001, 30, 1425-1428.

19. Hannapel, T.; Visbeck, S.; Knorr, K.; Mahrt, J.; Zorn, M.; Willig, F. Preparation of P-rich InPsurfaces via MOCVD and surface characterization in UHV. Appl. Phys. A 1999, 69, 427-431.

20. Vogt, P.; Frisch, A.M.; Hannapel, T.; Visbeck, S.; Willig, F.; Jung, C.; Esser, N.; Braun, W.; Richter, W. Atomic surface structure of MOVPE-grown InP(001). Phys. Stat. Sol. B 1999, 215, 737-742.

21. Muñoz, A.G.; Heine, C.; Lublow, M.; Klemm, H.W.; Szabó, N.; Hannappel, T.; Lewerenz, H.-J. Photoelectrochemical conditioning of MOVPE p-InP films for light-induced hydrogen evolution: Chemical, electronic and optical properties. ECS J. Solid State Sci. Techn. 2013, 2, Q51-Q58.

22. Wager, J.F.; Geib, K.M.; Wilmsen, C.W.; Kazmerski, L.L. Native oxide formation and electrical instabilities at the insulator/InP interface. J. Vac. Sci. Technol. B 1983, 1, 778.

23. Döscher, H.; Supplie, O.; May, M.M.; Sippel, P.; Heine, C.; Muñoz, A.G.; Eichberger, R.; Lewerenz, H.J.; Hannappel, T. Epitaxial III-V films and surfaces for photoelectrocatalysis. ChemPhysChem 2012, 13, 2899-2909.

24. Bertness, K.A.; Kurtz, S.R.; Friedman, D.J.; Kibbler, A.E.; Kramer, C.; Olson, J.M. 29.5\%efficient GaInP/GaAs tandem solar cells. Appl. Phys. Lett. 1994, 65, 989-991.

25. Guter, W.; Schone, J.; Philipps, S.P.; Steiner, M.; Siefer, G.; Wekkeli, A.; Welser, E.; Oliva, E.; Bett, A.W.; Dimroth, F. Current-matched triple-junction solar cell reaching $41.1 \%$ conversion efficiency under concentrated sunlight. Appl. Phys. Lett. 2009, 94, 223504.

26. Deutsch, T.G.; Head, J.L.; Turner, J.A. Photoelectrochemical characterization and durability analysis of GaInPN epilayers fuel cells and energy conversion. J. Electrochem. Soc. 2008, 155, B903-B907.

27. Letay, G.; Bett, A.W. A program for calculating limiting efficiency and optimum bandgap structure for multi-bandgap solar cells and TPV cells. Proc. 17th EU PV Sol. En. Conf. 2001, 178181. 
28. Maier, S.A.; Atwater, H.A. Plasmonics: Localization and guiding of electromagnetic energy in metal/dielectric structures. J. Appl. Phys. 2005, 98, 011101.

29. Dragoman, D.; Dragoman, M. Optical Characterization of Solids, Springer Verlag, Berlin, 2002.

30. Quinten, M.; Leitner, A.; Krenn, J.R.; Aussenegg, F.R. Electromagnetic energy transport via linear chains of silver nanoparticles. Opt. Lett. 1998, 23, 1331-1333.

31. Nakayama, K.; Tanabe, K.; Atwater, H.A. Plasmonic nanoparticle enhanced light absorption in GaAs solar cells Appl. Phys. Lett. 2008, 93, 121904.

32. Chang, T.H.; Wu, P.H.; Chen, S.H.; Chan, C.H.; Lee, C.C.; Chen, C.C.; Su, Y.K. Efficiency enhancement in GaAs solar cells using self-assembled microspheres. Opt. Express 2009, 17, 6519-6524.

33. Brus, L. Noble metal nanocrystals: Plasmon electron transfer photochemistry and single-Molecule Raman spectroscopy. Acc. Chem. Res. 2008, 41, 1742-1749.

34. Adams, M.W.W. The structure and mechanism of iron-hydrogenases. Biochim. Biophys. Acta, Bioenerg. 1990, 1020, 115-145.

35. Frey, M. Hydrogenases: Hydrogen-activating enzymes. ChemBioChem 2002, 3, 153-160.

36. Oudart, Y.; Artero, V.; Pécaut, J.; Fontecave, M. [Ni(xbsms)Ru(CO)2Cl2]: a bioinspired nickel-ruthenium functional model of [NiFe] hydrogenase. Inorg. Chem. 2006, 45,4334-4336.

37. Barton, B.E.; Matthew Whaley, C.; Rauchfuss, Th.B.; Gray, D.L. Nickel-iron dithiolato hydrides relevant to the [NiFe]-hydrogenase active site. J. Am. Chem. Soc. 2009,131, 6942-6943.

38. Hiraishi, T.; Kamachi, T.; Okura, I. Photoinduced hydrogen evolution with viologen-linked ruthenium(II) complexes and hydrogenase. J. Mol. Cat. A: Chem. 1999, 138, 107-113.

(C) 2014 by the authors; licensee MDPI, Basel, Switzerland. This article is an open access article distributed under the terms and conditions of the Creative Commons Attribution license (http://creativecommons.org/licenses/by/3.0/). 